\title{
Protée
}

\section{Éléments pour une biographie du Groupe $\mu$}

\section{Sémir Badir}

Volume 38, numéro 1, printemps 2010

Le Groupe $\mu$ entre rhétorique et sémiotique

URI : https://id.erudit.org/iderudit/039698ar

DOI : https://doi.org/10.7202/039698ar

Aller au sommaire du numéro

Éditeur(s)

Département des arts et lettres - Université du Québec à Chicoutimi

ISSN

0300-3523 (imprimé)

1708-2307 (numérique)

Découvrir la revue

Citer cet article

Badir, S. (2010). Éléments pour une biographie du Groupe $\mu$. Protée, 38(1), 9-18. https://doi.org/10.7202/039698ar

\section{Résumé de l'article}

Comme aujourd'hui les politiques de la recherche mettent en valeur le travail collectif et interdisciplinaire, le parcours intellectuel du Groupe $\mu$, long et étoffé, est susceptible d'être pris en exemple. Pour commencer, la présente étude éclaire les années de formation et d'identification du groupe, à la fin des années 1960. Elle retrace ensuite la réflexion parcourue en délimitant quatre périodes dans la chronologie des publications. Finalement, elle interroge les caractéristiques épistémologiques d'une recherche collective dans un secteur - celui de la nouvelle rhétorique - que cette recherche a contribué, pour une large part, à définir, mettant en avant ses positionnements programmatiques, gnoséologiques et paradigmatiques. 


\section{ÉLÉMENTS POUR UNE BIOGRAPHIE DU GROUPE $\mu$}

SÉMIR BADIR

Au moment d'entreprendre une biographie du Groupe $\mu$, quelques doutes surviennent sur la conduite à mener. Comment fait-on la biographie d'un groupe? Une biographie, comme son nom l'indique, est liée à une vie. Mais, assigner une vie à un groupe, n'est-ce pas avaliser d'emblée une métaphore? Ça tombe bien, pourrait-on dire, nous sommes en pays de connaissances. Pourtant, le travail de Groupe $\mu$ n'est pas de cultiver les métaphores, mais bien de les disséquer afin de savoir d'où vient leur efficace.

Du reste, les modèles «biographiques», concernant les activités de groupe, ne s'appliquent guère au nôtre et ne sont d'ailleurs pas si nombreux qu'on aurait pu l'imaginer. On sait que le Groupe $\mu$ aime à se comparer à Bourbaki ${ }^{1}$. Mais, en termes de parcours biographique, les différences sautent aux yeux. Car Bourbaki a un prénom, il est né d'un père et d'une mère; il existe même un faire-part de mariage de sa fille Betti avec l'administrateur délégué de la société des structures induites ${ }^{2}$ !

Quant à l'histoire littéraire, elle regorge de groupes, elle aussi, qu'elle catalogue dûment comme tels - groupe du Lundi, groupe surréaliste, groupe Tel Quel -, mais, en comparaison, la longévité du Groupe $\mu$ demeure un fait remarquable: 40 ans (19702010), cela dépasse très largement la durée de vie des meilleurs groupes littéraires, et il faut d'ailleurs revoir à la hausse cette évaluation de l'âge du Groupe $\mu$, qui ne correspond qu'à son âge public. En outre, la notoriété des groupes littéraires n'est mesurée qu'à l'aune de celle de ses membres, ce qui laisse entendre la prévalence de ceux-ci sur l'identité collective. Or, sans vouloir préjuger de la notoriété personnelle des membres du Groupe $\mu$, c'est bien un collectif qui est visé ici, et non une collection d'individus.

\section{LES ANNÉES DE FORMATION}

On pourrait alors faire commencer la biographie du Groupe $\mu$ par les premières rencontres entre ses membres. Les souvenirs des uns et des autres permettent d'établir que la première réunion du Groupe (qui ne s'appelait évidemment pas encore Groupe $\mu$, voire n'avait même pas encore conscience d'être un groupe) eut lieu à l'été 1963, en dehors de l'institution universitaire. Quatre des signataires de Rhétorique générale y étaient présents ${ }^{3}$. Pourquoi s'étaient-ils réunis? Pour se dire à eux-mêmes ce qu'ils avaient de commun, malgré la disparité de leurs activités professionnelles et de leurs domaines de spécialités. Or, ce qu'ils avaient de commun, outre d'être nés dans 
les années $1930^{4}$, c'est d'être des hommes épris de modernité, en particulier - mais pas exclusivement -, dans le champ de la littérature et des études littéraires.

Ils s'étaient reconnus ce goût-là à l'occasion de réunions auprès d'autres associations savantes nouvellement créées - on pense ici surtout à la Société internationale de symbolisme dirigée par Claire Lejeune ${ }^{5}-$, ou d'autres équipes littéraires - par exemple, celle du Cercle interfacultaire de littérature de l'Université de Liège animée par Arsène Soreil, ou celle du Journal des poètes. Ils avaient vu ce goût commun renforcé par des aversions non moins communes ainsi que par des ambitions partagées, comme cela se comprend bien, compte tenu de leur positionnement au sein de l'institution - à plusieurs, on se sent évidemment plus forts, tels des «chrétiens des catacombes», mais en version joyeuse, précise tout de même Jacques Dubois, fomentant «une terrible machine de guerre contre la vieille université» ${ }^{6}$. Bref, en cet été 1963, ces trentenaires tout feu tout flamme forment ce que les sociologues appellent, depuis Max Weber, une "communauté émotionnelle», quoiqu'en l'occurrence il n'y eut pas besoin de prophète pour les rassembler.

Qui sont-ils ces hommes qui se feront reconnaître, quelques années plus tard, comme le Groupe $\mu$ ? Il y a Jacques Dubois, à qui est attribuée l'initiative de la réunion et qui était alors chercheur au Fonds national de la recherche scientifique (FNRS), au Service de littérature française des XIXe et XXe siècles; Philippe Minguet, également chercheur au FNRS, mais au Service d'esthétique et qui, pour sa part, occupe le rôle de "grand planificateur"; ensuite, Francis Edeline, ingénieur chimiste formé à la Faculté universitaire des sciences agronomiques de Gembloux, petite ville située entre Wavre et Namur, et presque loin de Liège ${ }^{7}$; enfin Hadelin Trinon, animateur d'un cinéclub dans l'agglomération liégeoise et qui se fera connaître l'année suivante par des entretiens avec Andrzej Wajda (1964) ${ }^{8}$. À ces quatre-là s'adjoindront donc, quelques années plus tard, Francis Pire, assistant au Service de psychologie, quand la psychologie n'était pas encore organisée en une faculté à part entière, mais constituait une simple discipline adjacente à la philosophie; et Jean-
Marie Klinkenberg, qui venait de finir sa licence en philologie romane, aussitôt enrôlé au Service de stylistique, et qui apparaît donc comme le benjamin de la bande (il a vingt-trois ans quand il rejoint le Groupe à la fin de l'année 1967). En somme, à l'exception de Francis Edeline, tous avaient à voir avec la «Romane», ainsi qu'on désignait, et qu'on désigne encore par facilité, le Département de philologie romane de l'Université de Liège, soit qu'ils y aient fait leurs études, soit qu'ils y soient rattachés par le poste occupé dans l'institution. Et tous, Francis Edeline le premier, étaient passionnés par la littérature, et par la poésie en particulier.

Mais revenons à l'été 1963. Si ladite réunion fut un moment fondateur, ce n'est pas parce que ces hommes se sont reconnus des goûts, des intérêts communs, mais bien parce qu'ils décident de ne pas en rester là. Ils entendent se mettre à un certain travail en commun et, pour cela, il faut bien qu'ils discutent d'un projet. C'est ici que le Groupe a rendez-vous avec l'Histoire. Car ce qui fédère la variété des compétences que ces hommes mettront en jeu dans un travail collectif, c'est le structuralisme. Le structuralisme, dont le nom n'est pas encore l'objet de toutes les ignominies, apparaît alors en effet comme le paradigme épistémologique de cette modernité que chacun des futurs membres du Groupe $\mu$, dans son domaine respectif, a l'ambition d'embrasser. Aussi le travail commun commence-t-il avec la lecture d'un texte, paru un an plus tôt (en 1962) dans une revue d'anthropologie: la fameuse analyse des «Chats» par Jakobson et Lévi-Strauss (1962)9. Rappelons - car cette lecture fut décisive pour les travaux du Groupe - que Jakobson et Lévi-Strauss définissent deux figures de rhétorique, nommément la métaphore et la métonymie, comme les matrices littéraires sur lesquelles peut s'articuler une double analyse structurale - respectivement, analyse paradigmatique et analyse syntagmatique. Certes, ce n'est pas le structuralisme qui décida de la réunion de ces hommes - ni le structuralisme ni aucun de ses représentants n'étaient le prophète parmi cette assemblée -, mais c'est bien lui et les références intellectuelles qu'il induit qui leur permirent de concrétiser leurs velléités communautaires. 


\section{LES ANNÉES D'IDENTIFICATION}

Une fois le Groupe $\mu$ mis au travail, il ne lui resta plus qu'à tirer profit de ce travail, mais, naturellement, ce n'est rien de le dire aujourd'hui, encore fallut-il le faire. Le processus d'identification qui permit de passer d'un travail collectif au travail d'un collectif s'établit en quelques jalons rapprochés, dans les années 1967-1968:

- La décision d'honorer collectivement la demande d'une conférence à la Société internationale de symbolisme; conférence intitulée «Rhétorique généralisée» qui sera prononcée à quatre voix le 5 mars 1967 et publiée l'année suivante dans les Cahiers internationaux de symbolisme (n॰ 15-16).

- Subséquemment, le choix d'un nom pour cette présentation collective - ce sera donc celui de Groupe $\mu$, la dixième lettre de l'alphabet grec ayant été choisie pour désigner la métaphore. Qu'on s'en avise, c'est par synecdoque et métonymie que la métaphore a pu servir de signe de ralliement: synecdoque, en effet, et plus exactement synecdoque particularisante de type $\Pi$, puisque le panel des figures est appelé à être représenté par le symbole d'une d'entre elles; mais aussi métonymie, l'agent étant désigné par l'objet; serait-ce donc que la métaphore est la reine des figures, si elle est ainsi capable de les plier toutes à son service?

- La présentation de nouvelles communications, en divers colloques, principalement en France et en Italie (Jean-Marie Klinkenberg à Besançon en avril 1968, Jacques Dubois à Nancy en mai 1968 [voir Badir, 2010]), Hadelin Trinon à Urbino en 1969), mais toujours annoncées comme le fruit d'un travail collectif.

- La publication, enfin, de Rhétorique générale, en janvier 1970 chez Larousse, dans la collection «Langues et langage» dont le prestige - le capital symbolique, s'il faut en parler comme les bourdieusiens - était alors à son faîte.

Le jalonnement de ce coming out fut rendu nécessaire par des raisons sociologiques, à savoir la situation périphérique du Groupe, dont la majorité des membres enseignent dans une université belge de langue française, et par la condition privée de ses statuts, le groupe ne songeant que très tardivement à se faire reconnaître par l'institution en tant que "Centre d'études poétiques de l'Université de Liège», mention que l'on trouve en page de titre de Rhétorique générale, mais qui ne s'imposera guère, ni pour les membres du Groupe ni pour leurs interlocuteurs, lesquels leur préférant la dénomination synthétique de "Groupe de Liège» - encore une manipulation rhétorique, mais qui n'a pas été sans effets, vu l'ambiance concurrentielle qui existait alors au sein de la Romane liégeoise ${ }^{10}$. À ces raisons sociologiques, il faut en ajouter d'autres plus spécifiques aux conditions pratiques de la diffusion des travaux universitaires, conditions qui ne favorisent guère la présentation collective: à l'oral, dans les colloques et les conférences, un groupe, cela coûte cher à inviter et cela prend du temps à s'exprimer; à l'écrit, apparaissent les difficultés de la référenciation bibliographique, difficultés que le groupe fait voir, avec l'humour qui le caractérise - un humour de rhétoriqueurs, bien entendu - en référençant euxmêmes Rhétorique générale, trop souvent cité sous la mention «J. Dubois et al.», comme un ouvrage écrit par «Jacques Dubois, Francis Dubois, Jean-Marie Dubois, Philippe Dubois, Francis Dubois et Hadelin Dubois». Dans la même veine humoristique, Jacques Dubois, à qui il était demandé quelques années plus tard comment se passe une réunion de travail du Groupe $\mu$, répondait: «Eh bien c'est simple: Philippe Minguet ouvre la porte et nous fait asseoir, Francis Edeline pense, Jean-Marie Klinkenberg rédige et moi je signe».

Après la sortie de Rhétorique générale, qui sera traduit dans une dizaine de langues, les participations aux colloques et aux congrès se multiplient - à Amsterdam, au Centre de linguistique et de sémiotique d'Urbino dès la première année des séminaires d'été (en 1972), au Mexique, à la Columbia University de New York (où les accueille Michael Riffaterre) en 1972, à Barcelone, à Palerme, au Congrès mondial d'esthétique de Bucarest en 1972, enfin au Premier congrès international de sémiotique, en 1974 à Milan. Le Groupe $\mu$, représenté par l'un ou l'autre de ses membres, est définitivement lancé. 
Si nous nous sommes attardés, au risque du fastidieux, sur les débuts, et même la préhistoire, du Groupe $\mu$, c'est que c'est à ce moment, surtout, que la biographie d'un groupe se démarque de celle d'un individu. De cette évocation, deux traits spécifiques à la biographie de groupe peuvent être soulignés.

Premièrement, un groupe n'acquiert son identité qu'à la suite de sa formation, laquelle peut durer. Ainsi le Groupe $\mu$ ne s'est-il pas appelé aussitôt «Groupe $\mu$ ». Cela fait une notable différence avec les individus, dont l'identité - nom et prénom - est attribuée dès la naissance. Des «années de formation", disons entre 1962 et 1967, ont donc pu être évoquées avant que soient retracées, de 1967 à 1970, les «années d'identification".

Deuxièmement, il est évident que cette biographie de groupe recoupe celle des hommes qui le constituent, avec cette différence, non moins évidente, mais c'est une complication supplémentaire pour le biographe, que le début biographique de ces hommes ne coïncide pas avec le début du groupe, même sur le plan de leurs biographies intellectuelles. Bien sûr, sur chacun d'entre eux, on aurait pu être plus disert.

\section{LE GROUPE EN QUATRE PÉRIODES}

Pour la suite, la vue d'ensemble sera privilégiée sur une présentation détaillée des travaux du Groupe $\mu$. On peut discerner quatre périodes, chacune représentable comme une courbe dont le faite signifie la parution d'un livre - encore que, pour la quatrième période, la contemporaine, le livre reste à venir. En se la représentant comme une courbe, on comprendra sans doute que chaque période antérieure couve encore sous celles qui lui sont postérieures et produit encore, çà et là, des reprises, des amendements, sinon de nouvelles réflexions.

Quatre périodes, donc, pour quatre grands chantiers, et il suffit presque de reprendre les titres des ouvrages pour les désigner:

- la rhétorique générale, depuis les débuts publics du Groupe en 1967;

- la rhétorique de la poésie, depuis 1972 11;

- la rhétorique de l'image, depuis $1976^{12}$;

- enfin, une rhétorique de la connaissance, depuis 1994.

\section{Une rhétorique générale}

Le projet de rhétorique générale, élaboré à six, gouverne tous les travaux suivants. La raison en est que ce projet est immédiatement assorti de celui, tenu aussi largement et indéfiniment que possible, des «Rhétoriques particulières». En 1970, dans le no 16 de Communications, paraissent d'ailleurs quatre grandes études, portant sur la figure de l'argot, les titres de films, la clé des songes et les biographies de ParisMatch. On peut bien évidemment entendre, dans les différents domaines de ces recherches particulières, les intérêts spécifiques que les membres du groupe investissent dans le projet, mais celui-ci reste sous la responsabilité morale du collectif.

Le projet de rhétorique générale trouve à se synthétiser sous la forme d'un tableau, c'est-à-dire qu'il prend la forme d'une analyse faisant croiser plusieurs critères, celui des opérateurs logiques en ordonnées, celui des dimensions sémiotiques (tant paradigmatiques que syntagmatiques) en abscisses. Une telle présentation, fondée sur une analyse formelle, détonne avec le fourre-tout de la rhétorique ancienne. La nouvelle rhétorique apparaît ainsi, avant tout, comme un essai de rationalisation et de formalisation de données antérieures. Mais elle ne se borne pas au commentaire de la rhétorique ancienne - ce que Roland Barthes avait proposé dans son aidemémoire sur "L'Ancienne rhétorique», paru en 1970 dans le même numéro de Communications que les "Rhétoriques particulières» du Groupe $\mu$, quoique le manuscrit en circulât depuis le séminaire donné par Barthes à l'École pratique des hautes études durant l'année universitaire 1964-1965. Avec Rhétorique générale, la nouvelle rhétorique des figures adopte la posture de la tabula rasa théorique afin d'appliquer à la rhétorique les concepts de la linguistique structurale.

\section{Une rhétorique de la poésie}

En effectuant une analyse rhétorique de la poésie, le Groupe $\mu$ ne remplit pas seulement le programme de ses rhétoriques particulières. Les enjeux d'une confrontation avec la poésie dépassent le cadre de la simple application. Au moment de la sortie de Rhétorique générale, l'opinion commune concernant 
la rhétorique, en tant que domaine de connaissances et discipline fondée sur ces connaissances, était que celle-ci trouve dans la poésie le vivier d'où peuvent s'extraire pour l'essentiel toutes les figures, de sorte que la rhétorique apparaissait comme une province des études littéraires. Il importait que le Groupe $\mu$ prenne position face à cette supposition commune, ne fût-ce que pour éclairer rétrospectivement le projet de sa rhétorique générale. Rhétorique de la poésie ${ }^{13}$ commence donc par redresser une visée directrice, répondre à des critiques, parer à des objections et avertir d'enjeux épistémologiques, parmi lesquels on retiendra, pour l'exemple et parce qu'il intervient dans la compétition qui se jouait alors entre les projets des différents chercheurs, celui de la distinction de la nouvelle rhétorique d'avec la poétique.

Si la poésie n'est pas une application du modèle exposé dans Rhétorique générale, ou si, à l'inverse, la rhétorique générale n'est pas seulement une modélisation théorique d'un aspect de la poésie, c'est parce qu'en cette rencontre il y a plus à faire qu'une description "formelle» 14 des figures dans la poésie; il faut rendre compte d'effets rhétoriques, ces effets consacrant la rencontre d'un mécanisme formel particulier, celui que décrit la rhétorique, avec les conditions sémantiques de la poésie. Le Groupe $\mu$ offre à cette occasion une lecture anthropologique. Cette lecture, dont la procédure fait elle-même l'objet de réflexions théoriques (le sous-titre de l'ouvrage Lecture linéaire, lecture tabulaire en apporte un témoignage immédiat), trouve là encore à se présenter sous un aspect graphique; il s'agit cette fois d'un schéma en triangle capable de présenter la structure sémantique de la poésie - de toute poésie, y compris la poésie lettriste (1984: 892), mais rien que d'elle.

\section{Pour une rhétorique de l'image}

Une rhétorique de l'image était établie au programme depuis les premiers travaux du Groupe. La présence de visualistes, tels Hadelin Trinon, spécialiste de cinéma, et surtout de Philippe Minguet, théoricien de l'art, la rendait d'emblée évidente. L'intérêt que témoigne le Groupe pour les images se fait d'ailleurs sentir très tôt, mais l'approche de ce corpus s'annonce délicate et ne se fait d'abord que par les marges - analyse de titres de films (voir "Rhétoriques particulières», 1970b), étude de poésie spatialiste (Edeline, 1977; Groupe $\mu$, 1995), études sur les collages (Groupe $\mu, 1978$ ), considérations théoriques sur le transfert des concepts tropiques de l'étude du langage verbal vers celui du langage visuel (Groupe $\mu$, 1976).

Pour une approche frontale, toutefois, il a fallu que le Groupe $\mu$ se résolve à amender considérablement le projet initial. De la même manière que la rhétorique du langage verbal s'est appuyée sur l'analyse structurale de ce langage, une rhétorique de l'image n'aurait de sens, aux yeux du Groupe, que si elle peut s'appuyer sur une analyse structurale d'un langage des images. Or, cette analyselà, le Groupe $\mu$, insatisfait des travaux existants en la matière (fussent-ils méritants), en ressent vivement le manque. Il s'est alors imposé la charge énorme de pourvoir au manque que son projet rhétorique lui faisait constater, sans évitement possible.

Une telle résolution aboutit avec un Traité $d u$ signe visuel - rien de moins - signé à trois, à savoir par les deux membres encore en activité, Francis Edeline et Jean-Marie Klinkenberg, ainsi que par le regretté Philippe Minguet ${ }^{15}$. Avec ce traité, l'identité disciplinaire du Groupe a connu une mutation importante, l'étiquette de sémioticien visualiste l'emportant désormais sur celle de rhétoricien.

\section{Une rhétorique de la connaissance}

Pourtant, avec le projet en cours, le Groupe $\mu$ montre que rien n'a compromis le projet initial. Au contraire, en préparant une rhétorique de la connaissance, ils confirment la portée générale de leur projet théorique: les concepts analytiques de la nouvelle rhétorique sont, selon eux, en relation étroite avec les concepts fondamentaux de la pensée. On attend avec impatience le résultat de leurs réflexions.

\section{QUELQUES TRAITS ÉPISTÉMOLOGIQUES}

DE L'EeUVRE DU GROUPE

Pour que cet aperçu ne se limite pas aux aspects événementiels, mais qu'il contribue à une biographie proprement intellectuelle, nous ferons, pour finir, 
quelques observations d'ordre épistémologique sur l'œuvre et le travail du Groupe $\mu$.

\section{Le programme}

Peut-être le secret de la longévité du Groupe tient-il dans le fait que, dès la parution de Rhétorique générale, il était fait état d'un programme. Ainsi qu'on en a dessiné le parcours, ce programme a présidé aux travaux publiés par le Groupe. Un tel programme n'était pas chose rare dans les années 1960. Dans ces années-là, en effet, on entendait parler du «programme saussurien» pour la sémiologie; Greimas avait un programme génératif et comptait sur une école pour le réaliser; et bien des auteurs attirés par les idées structuralistes éditèrent des tomes 1 , dont les tomes suivants, dans la plupart des cas, se font encore attendre. Dans le cas du travail collectif, la notion de programme présente deux avantages: premièrement, le programme permet de répartir le travail à faire entre les membres du Groupe et, deuxièmement, il engage le collectif sur un objectif à long terme. Ainsi, il paraît patent que le programme aura été un instrument épistémologique très efficace préparant au travail effectif du Groupe $\mu$.

\section{Discipline et interdiscipline}

Mais cela n'aura pas empêché, bien sûr, que le collectif se délite au fil du temps - d'autres forces sont entrées en jeu, faisant intervenir la dynamique psychologique des groupes, la sociologie des carrières universitaires, etc.: chaque membre individuel du Groupe $\mu$ a aussi une vie, et d'abord une vie intellectuelle, en dehors du Groupe, plus ou moins compatible avec la vie du Groupe. Une de ces forces capables de mettre en péril la cohérence du travail accompli au sein du collectif retient toutefois particulièrement l'attention; c'est la force gnoséologique, celle qui fait et défait les disciplines du savoir: la rhétorique était-elle de taille à maintenir l'identité du Groupe $\mu$ ? Le Groupe a exprimé à ce sujet des bribes de critique épistémologique et gnoséologique, concernant l'identité de la rhétorique, qui s'avèrent d'autant plus passionnantes à suivre qu'elles ont fluctué au cours des années. Dans un premier temps, certainement, la rhétorique lui est apparue comme une discipline en devenir, bien qu'elle ait eu aussitôt à essuyer deux assauts:

- l'assaut intérieur d'un concurrent, briguant la même étiquette pour mener la rhétorique dans une tout autre direction que le Groupe $\mu$ - il s'agit de la rhétorique de l'argumentation, initiée par Chaïm Perelman à l'Université Libre de Bruxelles; on ne reviendra pas sur cette lutte "entre Belges" (la disposition géographique renchérissant ici sur la position des campements gnoséologiques), laquelle a été souvent commentée ${ }^{16}$;

- un assaut depuis l'extérieur, par un voisin plus grand qu'elle et plus gourmand - la poétique. Sur la lutte aux frontières des disciplines, on remarque que le Groupe $\mu$ a opéré une série de manœuvres stratégiques: d'abord, le voisin Poétique sert d'allié à Rhétorique pour attaquer la vieille Stylistique ${ }^{17}$, au point que Jean-Marie Klinkenberg, au colloque de Cluny en 1968, prétende que la rhétorique s'attache à décrire la fonction poétique du langage (selon la catégorisation de Jakobson); plus tard, toutefois, il est dit que rhétorique et poétique, malgré leur alliance, ne doivent pas être confondues - ce qui, en clair, signifie que pèse une menace d'absorption de la rhétorique par la poétique - et, pour ne pas avoir l'air de se contredire, le Groupe entend qu' «on convien[ne] qu'il est préférable de ne pas appeler "poétique" la fonction ainsi nommée par Jakobson» (1977a: 3)!

La délicatesse de la position disciplinaire de la rhétorique a conduit le Groupe $\mu$ à songer à d'autres voies de reconnaissance. Dans un second temps - second d'un point de vue plus argumentatif que strictement chronologique -, la rhétorique a cherché à s'intégrer dans un domaine englobant, dans lequel elle pourrait accomplir sa destinée sans avoir à camper sur des positions disciplinaires. Le choix de ce domaine englobant, toutefois, s'est avéré au moins double et s'est rendu de ce fait problématique. Le choix le plus simple aurait été de ranger la rhétorique parmi les «sciences du langage». Hélas, dans les années 1970, cette configuration de savoirs n'était pas encore établie; n'existait alors que la linguistique, avec laquelle, prétend le Groupe $\mu$, 
[...] les rapports étaient nécessaires, et nécessairement tendus. Nécessaires: défniie comme une mise en oeuvre des moyens d'expression, la rhétorique devait faire son profit de l'étude $d u$ plus important de ces moyens d'expression. Tendus: la linguistique ayant dû sélectionner un objet prioritaire dans tous les phénomènes liés au langage et à son exercice, elle s'est d'abord donnée, à partir de l'indispensable dichotomie langue. parole, comme une science du code; restriction que ne pouvait se donner la rhétorique, science des discours. (Ibid.: 4)

Aussi le choix du Groupe $\mu$ se porte-t-il finalement, pour inscrire la rhétorique au sein d'un groupe disciplinaire plus large, sur la sémiotique. Plus large, la sémiotique? Oui, sans doute; en tout cas accueillante, puisqu'il ne s'agit pas d'affilier le Groupe $\mu$ à une école ou à un mouvement quelconque. En clair: le Groupe $\mu$ n'est ni greimassien, ni peircien - de toute façon, dans les années 1960 et 1970, le monde des sémioticiens paraissait moins scindé qu'il ne se présente aujourd'hui. Au sein même de la sémiotique, le Groupe $\mu$ pourra se tenir dans les marges, tel un outsider flamboyant. Quoi qu'il en soit, l'adhésion, au moins apparente, de la rhétorique à la sémiotique sera déterminante dans le parcours du Groupe $\mu$ et dans les inflexions qui seront données à son programme. Ainsi, le tournant visualiste, à la fin des années 1970, est-il consenti comme une contribution à la sémiotique, seule capable de fournir des fondements théoriques aux recherches dédiées à la rhétorique de l'image.

L'adhésion à la sémiotique ne semble d'ailleurs jamais être mise en question. Lisons par exemple l'argument gnoséologique sous-jacent à une question posée par le Groupe $\mu$ en 1976 à propos de l'objet complexe:

Qu'est-ce qu'un objet, qu'est-ce qu'un objet complexe? [...]

Tous ces thèmes ont déjà fait l'objet d'études, dont la plupart entendent se loger à l'enseigne de la sémiotique. Cependant, à nos yeux, ces travaux de sémiotique iconique pèchent souvent de deux façons. Certains d'entre eux se perdent [...] dans des abstractions spéculatives sur la représentation [...] et, à ce titre, ils relèvent davantage de l'esthétique que de la sémiotique.

L'argument gnoséologique avancé par le Groupe $\mu$ dans cette citation est donc le suivant: des travaux sur l'image se sont donnés pour sémiotiques alors qu'en réalité ils ont plus à voir avec l'esthétique. Est-ce à dire qu'il faudrait le leur faire admettre afin que les recherches sur l'image s'orientent plus résolument vers la pensée esthétique? Loin de là! Car l'esthétique est, semble-t-il, un lieu de perdition, où l'on ne produit que des abstractions spéculatives. Le sous-entendu se fait entendre d'autant plus fortement que parmi les signataires du Groupe $\mu$, rappelons-le, se trouve un professeur d'esthétique de notoriété internationale, Philippe Minguet. En fait, il faut au contraire que les travaux sur l'image entrent en meilleure adéquation avec leur enseigne. Ainsi, pour le Groupe $\mu$, la sémiotique est-elle bien l'horizon théorique des recherches rhétoriques. Dans cette perspective, on pourrait considérer que Jean-Marie Klinkenberg est devenu aussi le premier héritier des travaux du Groupe, lui qui occupe à l'Université de Liège une chaire de Rhétorique et sémiologie ${ }^{18}$.

Pourtant, l'autre "nouvelle rhétorique», celle qui se fait à Bruxelles, engagerait bien plutôt la rhétorique du côté de la philosophie, se rappelant que c'est un philosophe qui avait établi l'ancienne. Mais cette nouvelle possibilité pour l'inscription de la rhétorique n'est jamais considérée par le Groupe $\mu$. Un déni de la philosophie, s'accentuant d'ailleurs avec le temps, marque le profil des travaux du Groupe $\mu$.

Alors, dans un troisième temps, le Groupe $\mu$ songe pour la rhétorique à un statut parallèle à celui visé aujourd'hui par la sémiotique: non plus un statut disciplinaire, étant donné la généralité des propos tenus, et la versatilité des domaines abordés, mais un statut d'interdiscipline: un réseau de compétences théoriques et méthodologiques qui demeure transversal vis-à-vis des terrains particuliers des objets de connaissance. C'est pour le coup que le Groupe $\mu$ pense à valoriser la dimension collective de son travail, et fait valoir l'aspect intrinsèquement interdisciplinaire de ses recherches, lui qui, aujourd'hui, si l'on veut bien considérer les choses sous cet angle, réunit un littéraire et un chimiste.

\section{Les paradigmes}

Les deux aspects précédemment évoqués déterminent, pour une large part, la configuration 
épistémologique des travaux du Groupe $\mu$ : un aspect qui ne dépend que de lui, à savoir le caractère programmatique des recherches, et un aspect qui dépend de la situation de la rhétorique parmi les disciplines. Nous voudrions à présent en évoquer un troisième plus large encore, celui des paradigmes dans lesquels s'inscrivent ces travaux. La plupart du temps, la question des paradigmes est réglée en même temps que l'inscription disciplinaire. Un littéraire, par exemple, n'a pas vraiment de choix paradigmatique: son épistémè est, grosso modo, celle des humanités classiques; un physicien, tout de même, se pliera aux exigences de production du discours scientifique. Mais, pour le rhétoricien, comme pour le sémioticien d'ailleurs, l'inscription paradigmatique fait l'objet d'un véritable choix.

Là-dessus, on peut dire que le Groupe $\mu$ aura eu la volonté, tout au long de son parcours, de se rapprocher des conditions de production du discours en sciences dures. Au gré des modes en sciences humaines, le Groupe $\mu$ aura certes porté la bannière du formalisme structural, puis du pragmatisme, puis encore, à travers la psychologie des formes, du cognitivisme symbolique, «démarche dont on s'étonne», précise le Groupe $\mu$, «qu'elle ne se soit pas davantage imposée au sémioticien» (1976: 38); mais le désir de scientificité reste la ligne harmonique qui sous-tend chacun de ces accords provisoires. Par exemple, dès 1970, on trouve, dans un article consacré à l'argot, des courbes de Gauss, courbes de Poisson, profondeurs moyennes, tests de Pearson, tests $\chi$, etc., tous concepts mathématiques qui ne sont peut-être que le matériel de base du scientifique, mais dont on peut douter que les littéraires, lecteurs désignés de l'article en question, trouveront à faire quelque chose. À quoi attribuer ce désir de scientificité? Un commentateur, qui reprochait au Groupe $\mu$ son positivisme essentialiste (voir Kuentz, 1971; Badir, 2008), se voit répliquer que le travail a ordonné de commencer par là où le Groupe $\mu$ a commencé, qu'il ne tient qu'aux hommes de bonne volonté d'en poursuivre l'effort, et qu'on ne voit pas bien comment on procéderait autrement. Travail, effort, main à la pâte, pied à l'étrier et tête sur les épaules: plutôt que de voir dans les références aux sciences dures de simples effets rhétoriques de faire-valoir, ainsi que le feraient des Sokal et Bricmont, on peut estimer que la science correspond à l'idéal du Groupe $\mu$ : car, en principe, la science fait œuvre collective et anonyme, pour le bien public; elle résulte moins d'idées, telles qu'elles prendraient leur origine dans le génie de tel ou tel individu, que du travail expérimental de la pensée. Et, en l'occurrence, ce travail expérimental a porté ses fruits: trois ouvrages, trois directions de numéros spéciaux de revue, une bonne cinquantaine d'articles, à quoi il faut adjoindre au moins cent cinquante articles signés individuellement par l'un ou l'autre des membres du Groupe: cela n'est pas rien. Sans aucun doute, les sémioticiens sont redevables au Groupe $\mu \mathrm{du}$ succès de son expérience. 


\section{NOTES}

1. Entrevue dans P. F. Bundgaard et F. Stjernfelt (2009).

2. Qui voudrait délaisser la lecture de cet article pour prendre aussitôt un chemin de traverse et s'enquérir du canular qui a donné vie (et famille), sous le nom de Nicolas Bourbaki, à un illustre groupe de mathématiciens de la première moitié du XXe siècle, trouvera largement de quoi satisfaire sa curiosité sur la Toile, notamment à l'adresse: http://www.neverendingbooks.org/index.php/the-wedding-invitationthat-nearly-killed-andre-weil.html (page consultée le 23 février 2010).

3. Mais aussi au moins une autre personne: Paul Pieltain, assistant en Romane, auteur d'une thèse de stylistique sur Paul Valéry. Francis Pire et Jean-Marie Klinkenberg, quant à eux, rejoindront le Groupe quelques années plus tard. Rappelons qu'à l'été 1963, Jean-Marie Klinkenberg n'avait pas dix-neuf ans, puisqu'il est né en octobre 1944. 4. Par ordre de naissance: Hadelin Trinon (1929-1991), Francis Edeline (1930-), Philippe Minguet (1932-2007), Jacques Dubois (1933-) À ceux-ci s'adjoindront quelques années plus tard: Francis Pire (1939-) et Jean-Marie Klinkenberg (1944-).

5. Sous le parrainage de Gaston Bachelard, le premier numéro des Cahiers internationaux du symbolisme paraît en novembre 1962. Francis Edeline fera paraître, dans le second numéro (1963), une conférence, "Le symbole et l'image selon la théorie des codes", prononcée lors du colloque des 24-25 novembre 1962.

6. Tout le passage concernant le Groupe $\mu$ mérite d'être cité in extenso: «Philippe Minguet ne dérogeait pas à ce qui fait l'ordre académique mais à la condition de pouvoir y jouer sa partie sur les marges. [...] Qu'il ait réussi à faire installer ses bureaux dans un appartement off limits de la rue Magnette en dit long sur le souci qu'il avait de se tenir à distance critique. C'est là, par exemple, que le Groupe $\mu$ tint d'innombrables séances diurnes ou nocturnes. Nous comportant en chrétiens des catacombes, mais en version joyeuse, nous élaborions dans le secret du séminaire d'Esthétique - que quelques badauds prirent parfois pour une officine de soins de beauté - une terrible machine de guerre contre la vieille université. Il en sortirait une «rhétorique générale», œuvre de six idiosyncrasies fondues en une seule voix. [...] Philippe veillait jalousement à ce que notre petite communauté fasse bloc. Il en stimulait l'invention par sa drôlerie, ses trouvailles, son hospitalité. Il en excitait la productivité par ses impatiences. Souhaitions-nous peaufiner tel chapitre qu'il s'exclamait: allez, vite un petit schéma pour résumer le tout et j'envoie l'ensemble à Paris. Paris qui nous semblait imprenable. Heureusement, notre Rastignac veillait et son audace paya. Nous parûmes chez Larousse, qui donnait alors le ton en linguistique" (J. Dubois, 2007 : 105-106).

7. Le site de Louvain-la-Neuve, à proximité, n'existait pas encore. 8. À partir de 1967, Trinon enseigne à l'Institut national supérieur des arts du spectacle (I.N.S.A.S.), école de cinéma à Bruxelles, et s'affirme bientôt comme un infatigable animateur de toutes sortes d'activités autour du cinéma (radio, télévision, cinéclub, musée, administration du mécénat public, etc.).

9. Sur l'impact de cet article en guise de modèle structuraliste, voir la compilation de commentaires réalisée par Delcroix et Geerts (1981); on notera la présence, à titre de contradicteur, d'un article, originellement paru en 1968, de Paul Delbouille, enseignant dans le même département de Romane que Jacques Dubois et Jean-Marie Klinkenberg. Or c'est précisément une figure de contradicteur - le terme n'est pas désobligeant - que P. Delbouille fut aux yeux du Groupe $\mu$ pour leurs propres travaux.

10. «[...] à noter que cette antonomase - "groupe de Liège" - n'a guère été utilisée par nous, qui n'avons pas non plus l'habitude de désigner tel chercheur parisien comme "le fin poéticien de la rue de Tournon" " (dans l'Avant-propos de "Collages", Revue d'Esthétique, n 3-4 [1978:

10]). Encore une de ces précisions données par le Groupe $\mu$ dont la raison institutionnelle ne se donne à lire qu'entre les lignes, aussitôt complétée par un trait d'humour - mais qui sait encore que le séminaire de Roland Barthes à l'École pratique des hautes études se donnait rue de Tournon? La désignation de "groupe de Liège" fait tenir trop de place à Liège, où des collègues pourraient s'offusquer du monopole ainsi octroyé au Groupe $\mu$, mais, partout ailleurs, elle maintient le Groupe $\mu$ dans une place marginale, sinon marginalisée, non certifiée en quelque sorte. On comprend que le Groupe $\mu$ y soit attentif. Cependant, et pour tout dire, reconnaissons que lui-même peut entretenir l'équivoque lorsque, six années plus tard, on trouve sous sa plume une autodésignation en tant qu' "école de Liège" (dans "Avant-gardes et rhétoriques", 1984: 882).

11. La parution «imminente» de Rhétorique de la poésie est annoncée dès 1972 lors d'un séminaire à New York chez Michael Riffaterre. Par ailleurs, on trouve dans une étude sur un poème d'Éluard ("Rhétorique poétique: le jeu des figures dans un poème de P. Éluard», due encore aux six signataires de Rhétorique générale), parue en janvier 1972 dans les Documents de travail du Centre de sémiotique et de linguistique d'Urbino, une première ébauche de la triade Cosmos - Anthropos - Logos, triade qui va innerver la pensée théorique développée dans l'ouvrage paru cinq ans plus tard.

12. Suivant la date d'un article intitulé «"La chafetière est sur la table”. Éléments pour une rhétorique de l'image", paru dans le n²9 de Communication et langages au $1^{\text {er }}$ trimestre 1976. Un ouvrage sur la rhétorique des images est annoncé «en préparation» dès 1979 (dans "Rhétorique, sémiotiques", Revue d'Esthétique, nº 1-2 [1979: 180]). Cet ouvrage, comme on sait, s'intitulera en fin de compte Traité du signe visuel (1992), mais son sous-titre rend compte du projet initial: Pour une rhétorique de l'image.

13. Ainsi qu'un article paru en 1977 dans Poétique faisant un bilan sur Rhétorique générale. "Miroirs rhétoriques: Sept ans de réflexion» - c'est le titre de cet article - sera ajouté en postface à l'édition de poche de Rhétorique générale (Paris, Seuil, coll. «Points», 1982).

14. Le Groupe $\mu$ parle de description nucléaire. Deux autres niveaux descriptifs lui sont hiérarchiquement supérieurs: description autonome, qui ménage une place aux aspects sémantiques des figures, et description synnome, prenant en compte les considérations textuelles, génériques et discursives (1970a: 148-156).

15. Il faut constater que, en raison de son état de santé, Philippe Minguet ne participait plus activement aux recherches collectives depuis la parution du Traité du signe visuel.

16. Ici même par Michel Meyer, dans «Pour une théorie générale des figures", mais également par J.-M. Klinkenberg, dans son chapitre «Rhétorique de l'argumentation et rhétorique des figures: sœurs ou ennemies?» (1996)

17. À ce sujet, on se reportera utilement à l'article, ici même, de Madeleine Frédéric, «Les travaux du Groupe $\mu$ : amers pour la stylistique?».

18. La question de l'héritage et celle apparentée de la filiation restent problématiques en ce qui concerne les groupes intellectuels et artistiques. L'affiliation à l'automne 1976 de Philippe Dubois, alors assistant de Philippe Minguet, au Groupe $\mu$, abandonnée quelques années plus tard, a montré la difficulté d'intégrer de nouvelles recrues. La difficulté pointera à nouveau vers 1994, lorsqu'il s'agit pour le Groupe de démarrer le projet d'une rhétorique de la connaissance en invitant des chercheurs et de jeunes professeurs de l'Université de Liège à le rejoindre; l'invitation, hautement appréciée, parviendra à se concrétiser, mais sous la forme, plus conventionnelle, d'un groupe de discussion, le travail de pensée et d'écriture inhérent au Groupe $\mu$ restant le fait des seuls Francis Edeline et Jean-Marie Klinkenberg. Sur la page de Wikipédia dédiée au Groupe, la formule choisie pour aménager une place à ce groupe de discussion est celle des 
"membres associés", à quoi sont ajoutés également des «membres correspondants ": "Ses membres associés sont ou ont été Sémir Badir, Laurence Bouquiaux, Marcel Otte, Jean Winand, Bénédicte Vauthier, Philippe Dubois. Le Groupe compte également des membres correspondants comme Árpád Vigh ou Göran Sonesson " (http:// fr.wikipedia.org/wiki/Groupe_ $\mu$ [page consultée le 25 janvier 2010]). On notera dans la liste des membres associés la présence de Philippe Dubois, lequel a néanmoins cosigné plusieurs articles référencés dans la bibliographie du Groupe $\mu$. Par ailleurs, dans un esprit d'actualisation, cette liste pourrait être complétée par les noms de Véronique Servais et Maria Giulia Dondero. Il serait également utile de répertorier les "membres visiteurs", mais la liste s'allongerait alors considérablement.

\section{RÉFÉRENCES BIBLIOGRAPHIQUES}

BADIR, S. [2008]: «En altérant la rhétorique», dans S. Badir et J.-M. Klinkenberg (dir.), Figures de la figure. Sémiotique et rhétorique générale, Limoges, Pulim, 167-182.

[2010]: «Le Groupe $\mu$ et la théorie littéraire ", Actes du colloque "Cluny 40 ans après " (2007), Besançon, Presses universitaires de Franche-Comté.

BundgaARD, P. F. et F. Stjernfelt (dir.) [2009]: Signs and Meaning: Five Questions, New York, Automatic Press /VIP.

DelcroiX, M. et W. GeErTs [1981] : Les " Chats" de Baudelaire: Une confrontation de méthodes, Namur, Presses universitaires de Namur. Dubois, J. [2007]: «In Memoriam Philippe Minguet (1932-2007) » ArtEFact, no 26, 105-106.

EdELINE, F. [1977] : Ian Hamilton Finlay. Gnomique et gnomonique, Paris, Hazan.
Groupe $\mu$ [1970a]: Rhétorique générale, Paris, Larousse ;

[1970b]: «Rhétoriques particulières (Figure de l'argot, Titres de films, La clé des songes, Les biographies de Paris-Match", dans AA.VV., Recherches rhétoriques, numéro spécial de Communications, n $16,70-124$. — [1976]: "La chafetière est sur la table". Éléments pour une rhétorique de l'image", Communication et langages, n²9, 36-49.

[1977a]: "Miroirs rhétoriques: Sept ans de réflexion ", Poétique, no 29, 1-19;

1977b]: Rhétorique de la poésie. Lecture linéaire, lecture tabulaire, Bruxelles, Éd. Complexe;

[1978] «Douze bribes pour décoller (en 40000 signes)», Revue d'esthétique ("Collages"), n 3-4, 11-41;

[1984]: «Avant-gardes et rhétoriques", Les Avant-gardes littéraires au XXe siècle, vol. II, Budapest, Akademiai Kiado;

[1992]: Traité du signe visuel. Pour une rhétorique de l'image, Paris, Seuil, coll. "La Couleur des idées";

[1995] : «Un signe poético-pictural. Relecture d'un poème de Ian Hamilton Finlay", Signe poétique, signe pictural, numéro spécial de Créis, no 3, 79-101.

JakOBSON, R. et C. Lévi-Strauss [1962]: «"Les Chats” de Charles Baudelaire", L'Homme, II-1, 5-21.

KLINKENBERG, J.-M. [1996]: «Rhétorique de l'argumentation et rhétorique des figures: sœurs ou ennemies? ", 7e leçon de Sept leçons de sémiotique et de rhétorique, Toronto, Gref.

KuENTZ, P. [1971]: «Rhétorique générale ou rhétorique théorique», Littérature, n०4, 108-115.

Trinon, H. et W. Wajda [1964]: Propos de Wajda, Paris, Seghers. 